der Verfälschung zurück. Es empfiehlt sich dann, die Zusammensetzung der Mineralstoffe und Stickstoffverbindungen eingehend zu prüfen. Die qualitativen Reaktionen sind mit zur Beurteilung heranzuziehen; doch ist ihr Ausfall allein nicht maßgebend. Der erfahrene Chemiker wird es auch nicht unterlassen, die äußere Besohaffenheit der Säfte, Geruch und Geschmack bei der Beurteilung zu verwerten.

Die Gesichtspunkte, welche bei der Beurteilung der Konservierungsmittel in Frage kommen, sind unter anderen aus dem Gutachten der Königlichen Wissenschaftlichen Deputation für das Medizinalwesen ${ }^{1}$ ), betreffend die Verwendung von Salicylsijure zur Konservierung von Nahrungs- und Genußmitteln vom 8. Februar 1908, zu erseben. Als Konservierangsmittel wird in letzter Zejt außer Salicylsäure häufig Ameisensäure angetroffen.

Aus dem pharmakologischen Institut der Universitat Leipzig.

\title{
Ueber Verbindungen des Wismuts mit einigen aliphatischen Oxysäuren.
}

Von Dr. Hans Telle, Korps-Stabsapotheker beim XIX. Armeekorps.

(Eingegangen den 25. VII. 1908.)

Von den Verbindungen des Wismuts mit den wichtigsten Oxysäuren sind nur diejenigen der Weinsäure und Zitronensäure eingehender bearbeitet worden. Die Milchsäureverbindungen sind bisher nur sehr wenig umfangreich, und diejenigen der Apfelsäure meines Wissens nach überhaupt noch nicht beschrieben worden. Die Untersuchungsresultate der Weinsäureverbindungen des Wismuts widersprechen sich in vielen Punkten derart, daß es mir lohnend erschien, einerseits die bisherigen neueren Angaben und Resultate eingehender zu prüfen, andererseits die Verbindungen des Wismuts mit den Oxysäuren weiter zu erforschen.

Bevor ich auf die Verbindungen des Wismuts mit den Oxysäuren selbst eingehe, erlaube ich mir einige Untersuchungsresultate über das Wismuthydroxyd vorauszuschicken, wozu mich die An-

1) Ztschr. f. Unters. d. Nahr.- u. Genußm. 1908, 15, 440-445. 
gaben von G. $B$ a $\left.u d r a n^{1}\right)$ veraniaßt haben. Dieser Autor behauptet in seiner Studie über Brechweinsteine (Emétiques), daB es durchaus nicht gleichgültig sei, ob man beim Fällen des Wismuthydroxyds das Alkali in die Wismutnitratlösung oder die Wismutnitratlösung in das Alkali bringt. B a ud ra n kam so zu zwei Wismuthydroxyden, die sich durch ihren Wassergehalt und durch ihre Löslichkeit in Kaliumglyzerin unterscheiden sollen. Das eine Hydroxyd war in diesem Lösungsmittel leicht löslich, das andere unlöslich. $\mathrm{B}$ a u d $\mathrm{r}$ a $\mathrm{n}$ sprach nach seinen Untersuchungen, die durch analytische Daten nicht erhärtet werden, das eine Hydroxyd als Typus $\mathrm{Bi}(\mathrm{OH})_{3}$, das andere als $\left(\mathrm{BiO}[\mathrm{OH}]_{2}=\right.$ $\mathrm{Bi}_{2} \mathrm{O}_{3}+\mathrm{H}_{2} \mathrm{O}$ an.

Dem dreiwertigen Wismut entspricht das Wismutorthohydraxyd $=\mathrm{Bi}(\mathrm{OH})_{3}$, dessen quantitative Ausfällung aus Wismutnitrat oder Wismutchloridlösung nicht nur durch fixes Alkali, sondern auch durch srhwächere Basen, wie Ammoniak, geschehen kann. Erhitzt man die alkalische Lösung mit dem allsgefällten Hydroxyd zum Sieden, so geht dasselbe in das Wismutoxyd $\mathrm{Bi}_{2} \mathrm{O}_{3}$ über. Thib a $\mathbf{l}^{2}$ ) hat nachgewiesen, daß aus einer Wismutnitratlösung beim Fällen mittelst Hydroxylionen kein reines Hyd̉roxyd von der Formel $\mathrm{Bi}(\mathrm{OH})_{3}$ entsteht, sondern ein Gemenge mit den Oxysalzen der in Frage kommenden Säuren resultiert. Thiba ult schlägt zur Gewinnung eines vollkommen reinen Hydroxyds vor, das Wismutsalz in wasserhaltigem Glyzerin zu lösen, mit Kalilauge im Ueberschuß zu fällen und das freie Alkali mittelst Schwefelsäure zu nentralisięren. Es entsteht auf diese Weise ein vollkommen reines Wismuthydroxyd, das mit Wasser aufgeschwemmt ein Gallert darstellt. - Die Wismuthydroxyde, welche ich zu meinen Untersuchungen verwendet habe, sind nach den folgenden allgemein üblichen Fällungsmethoden dargestellt.

Sowohl das Hydroxyd nach $T h i b$ a $l t$, als auch das gewöhnliche, gehen beim Trocknen bei $105^{\circ}$ unter Verlust von einem Molekül Wasser in das Metahydroxyd BiOOH über. Glüht man nun das konstant bei $105^{\circ}$ getrocknete BiOOH über freier Flamme, so gehen zwei Moleküle BiOOH unter Abgabe von einem Molekül Wasser über in ein Molekül $\mathrm{Bi}_{2} \mathrm{O}_{3}$.

A r p p e hat diesen Vorgang analytisch bewiesen, indem er durch Glühen von 0,597 des bei $100^{\circ}$ getrockneten Hydroxyds, $0,5755 \mathrm{Bi}_{2} \mathrm{O}_{3}$ erhalten hat, was $3,60 \%$ Wasserverlust entspricht

1) G. B a u d r a n, Annal. Chim. Physic. (7). 19, 536-574, 1900.

2) Th i b a a lt, Journ. Pharm. Chim. (6), 12, 559, 1900.

3) A r p p e, Popp. Annal. 64, 237. 
und mit der berechneten Wassermenge von einem Molekül übereinetimmt.

Für meine Cintersuchungen mußte ich mir das Wismuthydroxyd erstens durch Eingießen einer Wismutnitratlösung in überschüssige Natronlauge, zweitens durch Hinzufügen von überschüssiger Natronlauge zu einer Wismutlösung darstellen, da ich beweisen will, daß es für die resultierenden Hydroxyde vollkommen gleich ist, ob das Alkali zur Wismutlösung oder die Wismatlösung zum Alkali hinzugefügt wird.

Hauptbedingung bei den zwei Fällungsmethoden ist die, daß in jedem Falle die gleichen Bedingungen, das heißt, gleiche Konzentration und Temperatur, eingehalten werden.

$\mathrm{Zu}$ diesem $\mathrm{Zwecke}$ löste ich Wismutsubnitrat in der gerade ausreichenden Menge kalter Salpetersäure auf, kühlte die Lösung möglichst unter $5^{\circ}$ ab und goß dieselbe in eine ebenfalls abgekühlte Natronlauge. Ein bedeutender UeberschuB von Alkali ist tunliohst zu vermeiden. Das entstandene Wismuthydroxyd wurde zuerst des öfteren mit destilliertem Wasser dekantiert und sohließlich auf dem Filter solange mit Wasser ausgewaschen, bis der Ablauf neutral reagierte. Als Kriterium der Reinheit des Wismuthydroxyds kann auch das Fehlen der Salpetersüurereaktion dienen. - Das vollkommen ausgewaschene Hydroxyd preßte ich des öfteren zwischen Filtrierpapier ab und wiederholte dies solange, als das Hydroxyd noch Feuchtigkeit abgab, um es auf diese Weise möglichst schnell lufttrocken zu machen.

Einen anderen Teil des noch fouchten Wismuthydroxyds trocknete ich bei $105^{\circ}$, um aus dem Orthohydroxyd das Metahydroxyd zu erhalten. - Denn wie oben bereits erwähnt, verliert Wismutorthohydroxyd bei $105^{\circ}$ ein Molokïl Wasser. Obgleich diese Wasserbestimnung nicht exakt scin kann, da man im lufttrockenen Wismuthydroxyd keine Konstante erblicken darf, legte ich dennoch den Wasserverlust analytisch fest und kann konstatieren, daß es nicht gleich glückte, ein möglichst konstantes lufttrockenes Hydroxyd zu erhalten; teils enthält dasselbe $z u$ viel, teils, besonders nach längerem Liegen an der Luft, zu wenig Wasser. Ich halte das Trocknen durch immer wieder erneutes Fließpapier am vorteilhaftesten.

Die angestellten Analysen ergaben folgende Zahlen:

1. 1,2400 lufttrockenes $\mathrm{Bi}(\mathrm{OH})_{3}$ nahmen bei $105^{\circ} 0,0835 \mathrm{H}_{2} \mathrm{O}$ $a b$, entsprechend $6,73 \%$.

2. 1,2682 lufttrockenes $\mathrm{Bi}\left(\mathrm{OH}_{3}\right)$ nahmen bei $105^{\circ} 0,0782 \quad \mathrm{H}_{2} \mathrm{O}$ $a b$, entsprechend $8,16_{\%}^{\circ}$. 
Cefunden:

1. $\mathrm{H}_{2} \mathrm{O}=6,73 \%$

2. $\mathrm{H}_{2} \mathrm{O}=6,16 \%$
Berechnet Bit $\left.\mathrm{OH}_{3}\right)=\mathrm{H}_{8} \mathrm{O}+\mathrm{BiOOH}$ :

$\mathrm{H}_{8} \mathrm{O} \Rightarrow 6,96 \%$.

Das bei $105^{\circ}$ konstante Metahydroryd glühte ich über freier Flamme, und die Analysen bestätigen A r p p e'si) Angaben, dả zwei Moleküle desselben beim Uebergang zum Wismutoxyd ein Molekil Wasser verlieren, denn es vertoren:

1. 0,9225 des bei $105^{\circ}$ getrockneten $\mathrm{BiOOH} 0,0345 \mathrm{H}_{2} \mathrm{O}$, enteprechend $3,73 \%$.

2. 1,0004 des bei $105^{\circ}$ getrockneten $\mathrm{BiOOH} 0,0350 \quad \mathrm{H}_{2} \mathrm{O}$, entsprechend $3,49 \%$.

\section{Gefunden:}

1. $\mathrm{H}_{2} \mathrm{O}=3,7,7 \%$

2. $\mathrm{H}_{2} \mathrm{O}=3,49 \%$

Das andere Wismuthydroxyd wurde so dargestellt, daß ich eine ungefähr berechnete Menge Natronlauge in geringem Ueberschuß $B$ in abgekühlte Wismutnitratlösung mit einmal eintrug.

Im übrigen ist die Darstellung unter den oben beschriebenen Bedingungen auisgeführt worden. Die angestellten Analysen ergaben nachstehende Zahlen:

1. 1,1966 lufttrockenen $\mathrm{Bi}(\mathrm{OH})_{\mathbf{a}}$ verjoren bei $105^{\circ} 0,0859 \mathrm{H}_{\mathbf{4}} \mathrm{O}$ intsprechend $7,17 \%$.

2. 1,0212 lufttrockenen $\mathrm{Bi}(\mathrm{OH})_{2}$ verloren bei $105^{\circ} 0,0730 \quad \mathrm{H}_{2} \mathrm{O}$, entsprechend $7,14 \%$.

Gefunden:

$\mathrm{H}_{2} \mathrm{O}=7,17 \%, 7,14 \%$

Berechnet:

$\mathrm{H}_{2} \mathrm{O}=6,98 \%$.

Die Resultate der Analysen des zu'eiten Metahydroxydes waren nachstehende:

1. 1,0100 des bei $105^{\circ}$ getrockneten $\mathrm{BiOOH}$ verloren beirn Glühen $0,0400 \mathrm{H}_{2} \mathrm{O}$, entsprechend $3,93 \%$.

2. 1,0224 des bei $105^{\circ}$ getrockneten $\mathrm{BiOOH}$ verloren beim Glühen $0,0395 \mathrm{H}_{2} \mathrm{O}$, entsprechend $3,86 \%$.

\section{Gefunden:}

1. $\mathrm{H}_{2} \mathrm{O}=3,93 \%$

2. $\mathrm{H}_{2} \mathrm{O}=3,80 \%$

Durch obige Untersuchungsergebnisse ist es bewiesen, da $B$ ¿s bei der Darstellung für die resultierenden Hydroxyde gleichgültig ist, ob die Wismutlösung in das Alkali oder umgekehrt gebracht wird. Nach meiner Ansicht dürfte $B$ a u d $r a n$ in dem einen Hydroxyd, welches er als Typus $\mathrm{Bi}(\mathrm{OH})_{3}$ ansprach, das Ortho-

1) Arppe, l. c. 
hydroxyd, in dem anderen aber das Metahydroxyd gehabt haben, denn nur so lassen sich seine Behauptungen erklären. Was nun die Löslichkeit dieser beiden Orthohydroxyde in Kaliumglyzerin betrifft, so habe ich feststellen können, daß sich beide Orthohydroxyde frischgefällt in obigem Lösungsmittel vollständig und leicht lö̈en. Die Hydroxyde lösen sich auch im gewöhnlichen Glyzerin. Je länger die Hydroxyde an der Luft liegen bleiben, desto schwerer löslicher werden dieselben. Das Metahydroxyd ist im Glyzerin unlöslich. Auch die Löslichkeitsversuche sprechen dafür, daß $B$ a u d $\mathbf{r a n}$ in dem einen Hydroxyd, das Ortho-, in dem anderen das Metahydroxyd vor sich hatte.

\section{Verbindungen des Wismuts mit der Mllchsâure.}

Die ersten Versuche, Verbindungen des Wismuts mit der Milchsäure darzustellen, stammen von $H$. E $n$ g e $l$ h a r d t 1 ). Er erhielt Wismutlaktate durch Behandlung von Wismutkarbonat oder Wismuthydroxyd mit Milchsäure (erst beim Eindampfen der abfiltrierten Lösung schied sich ein Wismutsalz in feinen Nädelchen aus) oder zweckmäßiger, durch Mischung möglichst mit Wismutoxyd gesättigter Salpetersäure mit konzentrierter Natriumlaktatlösung. Es entsteht hierbei ein Krystallbrei aus Natriumnitrat und Wismutlaktat; aus der Lösung des Gemenges in möglichst wenig Wasser schieden sich krystallinische Krusten des Wismutlaktates ab. Aus den Analysen ergab sich die Formel $\mathrm{BiO}_{3} \mathrm{C}_{12} \mathrm{H}_{10} \mathrm{O}_{10}$ - nach jetziger Auffassung etwa $\mathrm{C}_{6} \mathrm{H}_{8} \mathrm{O}_{6} \mathrm{Bi}$. Das Salz löste sich in kaltem Wasser wenig, beim Kochen reichlich; erst beim Eindampfen der heiß bereiteten Lösung setzten sich wieder Krystallkrusten $a b$, die in wenig Wasser klar löslich sind; durch viel Wasser wird starke Trübung hervorgerufen. Eng e l ha r d t vermutet, daß das (neutrale) Laktat durch Kochen mit Wasser in ein in wenig Wasser lösliches $\mathbf{s}$ a $\mathrm{u}$ r e s und ein wasserunlösliches basisches Salz zerlegt wird. Wurde die oben angegebene, zur Darstellung des Salzes dienende Reaktion, in der Kochhitze ausgeführt, so entstand, wie Engel hardt annimmt, von vornherein unlösliches basisches Salz.

B r ün ing $g^{\ell}$ konnte nach dem von Engelhardt beschriebenen Verfahren das ,neutrale" Laktat nicht rein erhalten, gelangte aber durch Behandlung von Milchsäure mit Wismuthydroxyd (Eindampfen der filtrierten Lösung) za sandkörnerartigen

1) H. En g $\theta 1 \mathrm{~h}$ a $\mathrm{rd}$ t, Liebig's Ann. 65, 367, 1848.

2) A. B r ün in g, Liebig's Annal. 104, 195 (1857). 
Krystallen, die gleichfalls etwa der Formel $\mathrm{BiC}_{6} \mathrm{H}_{8} \mathrm{O}_{6}$ entsprechen. Seither ist meines Wissens über Wismutlaktate nichts mehr publiziert worden.

Meine eigenen Beobachtungen ergaben zunächst, daß sich frisch gefälltes Wismuthydroxyd ziemlioh reichlich in wässeriger Milchsäure auflöst. Wie aus nachstehenden Versuohsdaten ersiohtlich ist, erhielt ich (in Uebereinstimmung mit Eng el hardt) durch Digestion des Hydroxyds mit Milchsäture bei Wasserbadtemperatur ein wasserunlösliches Laktat.

$30 \mathrm{~g}$ des nach der oben beschriebenen Methode frisch gefällten Wismuthydroxyds, von dem noch anhaftenden Wasser durch $\mathrm{Ab}$ pressen zwischen Filtrierpapier befreit, übergoß ich mit 30,0 Milchsüure vom spezifischen Gewicht 1,21 und digerierte diese Mischung auf dem Wasserbade, bis nach kurzer Zeit vollkommene Lösung des Hydroxyds eingetreten war. Die an einem kühlen Orte beiseite gestellte klare Lösung erstarrte nach ca. 6 Stunden zu einem Krystallbrei. Die dem Salz anhaftende überschüssige Milchsäure wusoh ich zuerst mit Alkohol und zuletzt mit Aether aus. Das resultierende Wismutlaktat krystallisiert in rhombischen Täfelchen, die sowohl in kaltem, als auch in heißem Wasser kaum löslich, erst beim längeren Kochen aber unter teilweiser Zersetzung etwas gelöst wurden.

Lufttrocken bei $105^{\circ}$ erhitzt, verlor das Salz nichts an Gewioht, enthielt also kein Krystallwasser.

Bei der Elementaranalyse gaben:

1. 0,1792: $0,0450 \mathrm{H}_{2} \mathrm{O}$, entsprechend $2,79 \% \mathrm{H}$ und $0,1220 \mathrm{CO}_{2}$, entsprechend $18,56 \% \mathrm{C}$.

2. 0,1992: $0,0465 \mathrm{H}_{2} \mathrm{O}$, entsprechend $2,59 \% \mathrm{H}$ und $0,1342 \mathrm{CO}_{2}$, entsprechend $18,37 \% \mathrm{C}$.

$$
\begin{gathered}
\text { Gefunden: } \\
\mathrm{H}=\mathbf{2 , 5 9 \%} \\
\mathrm{C}=\mathbf{1 8 , 5 6 \%}
\end{gathered}
$$

Berechnet auf $\mathrm{C}_{6} \mathrm{H}_{6} \mathrm{O}_{6} \mathrm{Bi}$ :

$$
\begin{aligned}
& \mathrm{H}=\mathbf{2 , 3 5 \%} \\
& \mathrm{C}=18,70 \% .
\end{aligned}
$$

Das Wismut in dem Wismutlaktat bestimmte ich als Wismutoxyd, indem ich eine gewogene Menge des über Schwefelsäure von der anhaftenden Luftfeuchtigkeit befreiten Salzes vorsichtig verbrannte, den Glührüokstand mit Salpetersäure abrauohte, und das so entstandene Wismutnitrat zu Wismutoxyd bis zur Konstanz glühte.

Diese Resultate sind wio folgt: $54,26 \% \mathrm{Bi}$.

1. 0,6312 milchsaures $\mathrm{Salz}$ gaben $0,3820 \quad \mathrm{Bi}_{2} \mathrm{O}_{3}$, entsprechend

2. 0,8293 milchsaures Salz gaben $0,4995 \mathrm{Bi}_{2} \mathrm{O}_{3}$, entsprechend $54,01 \% \mathrm{Bi}$. 

Gefunden:
1. $\mathrm{Bi}=54,26 \%$
2. $\mathrm{Bi}=54,03 \%$

Berechnet auf $\mathrm{C}_{6} \mathrm{H}_{9} \mathrm{O}, \mathrm{Bi}$ :

$\mathrm{Bi}=54,02 \%$.

Diese. Resultate passen also gut zu der Formel $\mathrm{C}_{6} \mathrm{H}_{9} \mathrm{O}_{6} \mathrm{Bi}$, wonach sich ein Atom Wismut mit zwei Molekülen Milchsäure verbunden haben.

Läßt man nun aber Milchøäure bei gewöhnlicher Temperatur auf frisch gefälltes Wismuthydroxyd einwirken, so erhält man leicht und in beliebiger Menge eine gut krystallisierende was s e rlösli c he Verbindung des Wismuts mit Milchsäure.

Zu einer gut verrührten Mischung von 30,0 frisch gefälltem, noch feuchtem Wismuthydroxyd und 10,0 Wasser setzt man 30,0 Milchsäure hinzu und schüttelt kräftig durch. Dabei erwärmt sich die Mischung merklich, und nach kurzer Zeit hat sich der Brei von Wismuthydroxyd in der Milchsäure vollständig gelöst. Diese Lösung; längere Zeit beiseite gestellt, soheidet reiohlioh glänzende Büschel nadelförmiger Krystalle ab. Den Hauptanteil der überschüssigen Milchsäure entfernt man zuerst durch Abpressen zwischen Filtrierpapier und wäscht dann die Krystalle noch mit Aether aus. Die so von der überschüssigen Milchsäure befreiten prismatischen Nadeln lösten sich leicht, ungefähr im Verhältnis 1:10 im Wasser auf.

Die Krystalle geben an der Luft, im Exsikkator über Schwefelsäure und schließlich beim Trocknen bei $105^{\circ}$ reichlich Krystallwasser ab. Der Krystallwassergehalt schwankte, je nachdem die Präparate möglichst frisch, lufttrocken oder nach Iängerer Aufbewahrung an der Luft untersucht wurden, zwischen $17-25 \%$.

1. 0,6253 lufttrocken verloren bei $105^{\circ} 0,1288 \mathrm{H}_{2} \mathrm{O}$, entsprechend $20,59 \%$.

2. 0,6810 lufttrocken verloren bei $105^{\circ} 0,1396 \mathrm{H}_{2} \mathrm{O}$, entsprechend $20,49 \%$. $25,08 \%$.

3. 0,7195 lufttrocken verloren bei $105^{\circ} 0,1805 \mathrm{H}_{2} \mathrm{O}$, entsprechend

4. 0,6214 lüftrocken verloren bei $105^{\circ} 0,1538 \mathrm{H}_{2} \mathrm{O}$, entsprechend $24,75 \%$. $16,8 \notin \%$.

5. 0,6410 lufttrocken verloren bei $105^{\circ} 0,1080 \mathrm{H}_{2} \Theta$, entsprechend $16,55 \%$.

6. 0,6051 lufttrocken verloren bei $105^{\circ} 0,1002 \cdot \mathrm{H}_{2} \mathrm{O}$, entsprechend

Das bei $105^{\circ}$ bis zur Konstanz getrocknete Salz gab bei der Elementaranalyse dieselben Zahlen wie das vorher beschriebene unlösliche Salz und zwar: 
1. 0,1340 wasserfreie Substanz gaben $0,0320 \mathrm{H}_{2} \mathrm{O}$, entsprechend $2,65 \% \mathrm{H}$ und $0,0025 \mathrm{CO}_{2}$, enteprechend $18,82 \% \mathrm{C}$.

2. 0,1346 gaben $0,0346 \mathrm{H}_{2} \mathrm{O}$, enteprechend $2,84 \% \mathrm{H}$ und $0,0926 \mathrm{CO}_{2}$ entsprechend $18,76 \% \mathrm{C}$.

3. 0,1782 geben $0,0430 \mathrm{H}, \mathrm{O}$, enteprechend $2,67 \% \mathrm{H}$ und $0,1238 \mathrm{CO}_{2}$, enteprechend $18,94 \% \mathrm{C}$.

4. 0,1790 gaben $0,0400 \mathrm{H}_{2} \mathrm{O}$, enteprechend $2,48 \% \mathrm{H}$ und $0,1230 \mathrm{CO}_{2}$, enteprechend $18,74 \% \mathrm{C}$.
Gefunden :
$\mathbf{H}=\mathbf{2 , 4 8 \%}$
$\mathrm{C}=18,77 \%$
Berechnet auf $\mathrm{C}_{9} \mathrm{H}_{9} \mathrm{O}_{6} \mathrm{Bi}$ :

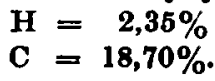

Das Wismut bestimmte ich in der bereits angegebenen Weise als Oxyd, die Resultate waren:

1. 0,4965 konstant bei $105^{\circ}$ getrocknetes Salz gaben 0,2992 $\mathrm{Bi}_{2} \mathrm{O}_{3}$, entsprechend $54,04 \% \mathrm{Bi}$.

2. $0,5414 \mathrm{Salz}$ gaben $0,3255 \mathrm{Bi}_{2} \mathrm{O}_{3}$, entsprechend $53,91 \% \mathrm{Bi}$.

3. 0,5330 Substanz gaben $0,3198 \mathrm{Bi}_{2} \mathrm{O}_{2}$ entspreahend $53,80 \% \mathrm{Bi}$.

4. 0,5049 gaben 0,3034 $\mathrm{Bi}_{2} \mathrm{O}_{3}$, entsprechend $53,88 \% \mathrm{Bi}$.
Gefunden:
$\mathrm{Bi}=54,04$
Berechnet auf $\mathrm{C}_{\mathrm{a}} \mathrm{H}_{9} \mathrm{O}_{\mathrm{a}} \mathrm{Bi}$ :
$\mathrm{Bi}=54,02 \%$.

Nach diesen Resultaten der Analyse ist anzunehmen, daß bei der Einwirkung von überschüssiger Milchsäure auf feuchtes, frisch gefälltes Wismuthydroxyd in der Kälte ein Hydrat des Wismutbilaktates $\mathrm{C}_{8} \mathrm{H}_{8} \mathrm{O}_{8} \mathrm{Bi}$ entsteht. Bei Zugrundelegung des höchsten beobachteten Gewichtsverlustes der Krystalle beim 'Trocknen auf $105^{\circ}$, der $24,75-25,08 \%$ betrug, berechnet sich der (iehalt des löslichen Bilaktates an Krystallwasser auf sieben Moleküle $\mathrm{C}_{6} \mathrm{H}_{9} \mathrm{O}_{6} \mathrm{Bi}+7 \mathrm{H}_{2} \mathrm{O}$.

Durch das Trocknen an der Luft und im Ersikkator verliert die Substanz allmïhlich ihre Löslichkeit in Waseor. Dio bei $105^{\circ}$ getrockneten Krystalle sind im Wasser ebensowenig löslich, wie das in der Wärme dargestellte krystallwasserfreie Bilaktat.

Die frisch bereitete Lösung des Hydrates in Wasser reagiert stark sauer und scheidet auf Zusatz von Sodalösung, ohne aufzubrausen, einen voluminësen, smorphen weißen Nioderschlag ab. Nach längerem Stehen trïbt sich dis Lösung allmählich schon bei gewöhnlicher Temperatur und setat einen körnig krystallinischen, aus undeutlioh entwickelten Rhomben bestehenden Bodensatz ab. Beim Kochen der konzentrierten Lösung erfolgt Trübung und Abscheidung des unlöslichen Körpers sofort. Engt man die Läsung im Exnikkator über Schwefelsäure langsam ein, so krystallisieren zunächsat die wohlansgebildeten Rhomben des krystallwasserfreion 
Wismutlaktates reichlich aus, zuletzt aber auch in geringerer Menge die langen Nadeln des Hydrates.

Hieraus erhellt, daß das lösliche Hydrat unbeständig ist und leicht in das unlösliche krystallwasserfreie Wismutlaktat umgewandelt wird. Hinsichtlich der Bedingungen, unter denen die Umwandlung stattfindet, wäre folgendes hervorzuheben.

1. Die Umwandlung erfolgt in der wässerigen Lösung des von überschüssiger Milchsäure befreiten reinen Hydrates bei gewöhnlicher Temperatur in geringerem Umfange von selbst, reichlicherer beim langsamen Verdunsten der Lösung über Schwefelsäure, ist aber auch im letzteren Falle keine vollständige; denn es scheiden sich (vergl. oben) zuletzt auch noch die nadelförmigen Krystalle des Hydrates aus.

2. Die Umwandlung wird verhindert durch Anwesenheit von freier Milchsäure in der Lösung. Es ergibt sich dies einerseits daraus, daß bei der Darstellung des Hydrates nach dem oben angegebenen Verfahren immer nur lösliches Hydrat erhalten wird, sowie auch aus den Daten des nachstehend mitgeteilten Versuches, bei welchem die mit verschiedenen Mengen freier Milchsäure versetate Lösung des Hydrates mit den Krystallen des Hydrates oder denjenigen des unlöslichen Wismutlaktates geimpft und die in der Kälte abgeschiedenen Krystallisationen untersucht wurden.

a) 1 Teil 10\% iger wässeriger Lösung des Hydrats versetzt mit 1 Teil Milchsäure und geimpft mit u n lö s li c hem Salz.

a) Dieselbe Lösung geimpft mit lö s l i c h e m Salz.

b) 2 Teile wässeriger Bilaktatlösung vermischt mit I Teil Milchsäure, geimpft mit u nlöslich e m Salz.

(3) Dieselbe Lösung mit löslichem Salz.

c) 4 Teile Bilaktatlösung vermischt mit 1 Teil Milchsäure, geimpft mit unlöslichem Salz.

y) Dieselbe Lösung mit löslichem Salz.

d) 9 Teile Wismutlaktatlösung mit 1 Teil Milohsäure vermischt, geimpft mit unlöslichem Salz.

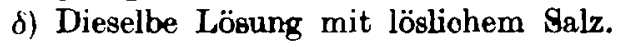

Aus sämtlichen Lösungen krystallisierte in der Kälte nur das lösliche Hydrat aus. (Unlösliches Salz wandelte sich durch Auflösen in freior Milchsäure nur langsam in das Hydrat um.)

3. Die Umwandlung wird sehr beschleunigt durch höhere Temperatur. Wie eingangs gezeigt wurde, entsteht bei der Einwinkung von Milchsäure auf Wismuthydroxyd bei Wasserbadtemperatur überhaupt nur krystallwasserfreies unlösliches Wismutlaktat. Weitere Versuche lehrten, daß sohon bei Temperaturen 
von $45-50^{\circ}$ die Umwandlung des Hydrates beginnt. Von der $\mathrm{k}$ a $\mathrm{lt}$ bereiteten Lösung von 20,0 frisch gefälltem Wismuthydroxyd, 10,0 Wasser und 20,0 Milchsäure wurden Proben von je $5 \mathrm{ccm}$ im Thermostaten auf $45^{\circ}$ erwärmt, und hierauf die eine mit den Krystallen des Hydrats, die andere mit denen des unlöslichen Wismutlaktates geimpft. Nach einer halben Stunde hatten sich in beiden Proben die rhombenförmigen Krystalke des unlöslichen Salzes abgeschieden.

Die Umwandlung erfolgt auch unter diesen Bedingungen allmählich, das heißt, sie ist von der Zeit abhängig, denn, als ich eine der Proben, in welcher sich bereits unlösliches Wismutlaktat abgeschieden hatte, aus dem Thermostaten entnahm und bei gewöhnlicher Temperatur stehen ließ, konnte ich konstatieren, daß allmählich neben den Rhomben auch die langen Nadeln des Hydrates auskrystallisierten. Bringt man auf $45^{\circ}$ vorgewärmte Milchsäure mit Wismuthydroxyd zusammen, so resultiert von Anfang an unlösliches Wismutlaktat.

$\mathrm{Da}$ B die Konzentration keinen merklichen Einflußß darauf hat, ob sich aus den kalt bereiteten Stammlösungen lö̈liches oder unlösliches Salz abscheidet, zeigt folgender Versuch:

\section{Ungeimpfte Lösungen.}

$\left.\begin{array}{l|c}\text { Stammlösung }\left(\mathrm{BiOH}_{3}+\mathrm{Milchsäure} \mathrm{ana)}\right. & \text { Auskrystallisiert } \\ 2 \mathrm{ccm} \text { Stammlösung }+8 \mathrm{ccm} \mathrm{H}_{2} \mathrm{O} \\ 1 \mathrm{ccm} \text { Stammlösung }+9 \mathrm{ccm} \mathrm{H}_{2} \mathrm{O}\end{array}\right\} \begin{gathered}\text { war nur die } \\ \text { lösliche Modifikation. }\end{gathered}$

Mi.t löslichem Salz geimpfte Lösungon.

Stammlösung

$2 \mathrm{ccm}$ Stammlösung $+8 \mathrm{ccm} \mathrm{H}_{2} \mathrm{O}$ Schied sich nur

$1 \mathrm{ccm}$ Stammiösung $\left.+9 \mathrm{ccm} \mathrm{H}_{2} \mathrm{O}\right\}$ lösliches Salz ab.

Nit unlöslichem Bilaktatgeimpfte Lösungen.

Stammlösung
$2 \mathrm{ccm}$ Stammlösung $\left.+8 \mathrm{ccm} \mathrm{H}_{2} \mathrm{O}\right\}$ Es resultierte nur

$1 \mathrm{ccm}$ Stammlösung $+9 \mathrm{ccm} \mathrm{H}_{2} \mathrm{O}$ die lösliche Form.

Zum Beleg dafür, daß das aus der Lösung des Hydrates durch Emwandlung entstandene unlösliche Salz identisch ist mit dem eingangs beschriebenen unlöslichen Wismutlaktat, folgen anbei die Daten der Analyse von Krystallen, die sich beim Einengen der Hydratlösungen im Exsikkator iiber Schwefelsäure abgeschieden hatten. 

$54,27 \%$ Bi.

0,6312 lufttrockener Substanz gaben 0,3820 $\mathrm{Bi}_{2} \mathrm{O}_{3}$, entsprechend

0,1855 gaben $0,0460 \mathrm{H}_{2} \mathrm{O}$, entsprechend $2,75 \% \mathrm{H}$ und $0,1260 \mathrm{CO}_{2}$, entsprechend $18,52 \% \mathrm{C}$.
Gefunden:
$\mathrm{H}=\mathbf{2 . 7 5} \%$
$\mathrm{C}=18,62 \%$
$\mathrm{Bi}=54,26 \%$
Berechnet $\mathrm{C}_{6} \mathrm{H}_{9} \mathrm{O}_{6} \mathrm{Bi}$ :
$\mathrm{H}=2,35 \%$
$\mathrm{C}=18,70 \%$
$\mathrm{Bi}=54,02 \%$.

Man konnte an die Möglichkeit denken, da $\beta$ die beiden von mir dargestellten Formen des Wismutbilaktates den Salzen racemischer resp. optisch aktiver Milchsäure entsprechen könnten. Es zeigte sich aber, daß, einerseits die $10 \%$ ige wässerige Lösung des Hydrates und andererseits die durch Zersetzung des unlöslichen Wismutlaktates mit Schwefelwasserstoff erhaltene freie Milchsäure, beide optisch inaktiv waren.

\section{Verbindungen des Wlsmuts mit der Apfelsäure.}

Analog der Darstellungsweise des milchsauren Wismuts versuchte ich frisch gefälltes Wismuthydroxyd kalt in einer wässerigen Apfelsäurelösung zu lösen. Da dieser Lösungsversuch nicht glückte, brachte ich fein mit Wasser verrührtes frisch gefälltes Wismuthydroxyd mit überschüssiger Apfelsäure zusammen und kochte die Mischung einige Minuten. Es resultierte eine klare sirupdicke Flüssigkeit, die sowohl in der Kälte, als auch bei kürzerem Stehen über Schwefelsäure keine Krystalle abschied. Nach längerem Stehen über Schwefelsäure erstarrte der Sirup zu einer weißen amorphen Masse, die sich klar im Wasser löste. Es ist trotz vieler Versuche nicht gelungen, aus diesem Sirup, der sehr viel freie Apfelsäure enthielt, irgend ein einwandfreies apfelsaures Wismut zu erhalten. Da man annehmen mußte, daß ein großer Ueberschuß von Apfelsäure störend auf die Darstellung des Wismutmalats einwirkte, mischte man 5,0 Wismuthydroxyd und eine Lösung von 10,0 Apfelsäure in wenig Wasser und kochte das Gemisch ca. 5 Minuten auf. Nach dem Absetzen und Erkalten der Mischung konnte man unter dem Mikroskop beobachten, daß der Niederschlag nicht aus amorphem Wismuthydroxyd bestand, sondern ein sphäroidisch krystallinischer Körper, aller Wahrscheinlichkeit eine Verbindung von Apfelsäure und Wismut, vorlag. Der weiß aussehende krystallinische Körper wurde auf ein Filter gebracht und die noch anhaftende Apfelsäure mit Wasser gut ausgewaschen. Das entstandene apfelsaure Wismut ist fast unlöslich in Wasser und wird durch fixes und kohlensaures Alkali zersetzt. 
Das lnfttrockene Salz gah, wie nachotehende Analyse bewiesen hat, beim Trocknen bei $105^{\natural} 5,14 \%$ Wasser ab.

1. 0,5477 verloren bei $105^{\circ} 0,0282 \mathrm{H}_{2} \mathrm{O}$, entsprechend $5,14 \%$.

2. 0,5240 verloren bei $105^{\bullet} 0,0275 \mathrm{H}_{2} \mathrm{O}$, entsprechend $5,24 \%$.

3. 0,5245 verloren bei $105^{\circ} 0,0273 \mathrm{H}_{2} \mathrm{O}$, entsprechend $5,20 \%$.

Das Wismut beabsichtigte ich wie bei der Milchsäure als Oxyd zu bestimmen, bin aber nach einer solchen Bestimmung von dieser Methode abgekommen, da das Wismutmalat beim Erhitzen über freier Flamme leicht explodiert und man aüßerst vorsichtig zu Werke gehen muß. Aus díssen Gründen löste ich eine abgewogene Menge in wenig Salzsäure, verdünnte mit Wasser und leitete bis zar Sättigung Schwefelwasserstoffgas ein. Das auf einen Goochschen Tiegel gebrachte Wismutsulfid wurde zuerst mit Alkohol, dann mit 8chwefelkohlenstoff und zuletzt wieder mit Alkohol gewaschen. Die Analysen gaben folgende Ergebnisse:

1. 0,5195 bei $105^{\circ}$ getrocknetes $\mathrm{Salz}$ gaben $0,3910 \mathrm{Bi}_{2} \mathrm{~S}_{3}$, entsprechend $61,15 \% \mathrm{Bi}$.

2. 0,4965 wasserfreie Substanz gaben $0,373 \mathrm{Bi}_{2} \mathrm{~S}_{3}$, entsprechend $61,04 \% \mathrm{Bi}$.

Ich lasse noch eine Wismutbestimmung als Oxyd folgen:

3. 0,4972 bei $105^{\circ}$ getrocknetes $\mathrm{Salz}$ gaben $0,3370 \mathrm{Bi}_{2} \mathrm{O}_{3}$, entsprechend $60,78 \% \mathrm{Bi}$.

Die vorstehenden Analysenzahlen stimmen gut mit der Formel $\mathrm{C}_{4} \mathrm{H}_{3} \mathrm{O}_{5} \mathrm{Bi}$ überein, und es muß sonach. eine Verbindung von einem Atom Bi mit einem Molekül Apfelsäure vorliegen.
Gefunden:
$\mathrm{Bi}=61,13^{\circ} \%$
Berechnet suf $\mathrm{C}_{4} \mathrm{H}_{3} \mathrm{O}_{5} \mathrm{Bi}$ :
$\mathrm{Bi}=61,35 \%$.

Berechnet man auf die Formel $\mathrm{C}_{4} \mathrm{H}_{2} \mathrm{O}_{5} \mathrm{Bi}$ ein Molekïl Wasser, so beträgt dasselbe $5,04 \%$.
Gefunden:
$\mathrm{H}_{2} \mathrm{O}=5,14 \%$
Berechnet auf $\mathrm{C}_{4} \mathrm{H}_{3} \mathrm{O}_{5} \mathrm{Bi}+1 \mathrm{H}_{2} \mathrm{O}$ :
$\mathrm{Bi}=5,04 \%$.

Die nachstehende Elementaranalyse bestätigt die Annahme obiger Formel.

0,1760 wasserfreie Substanz gaben $0,0230 \quad \mathrm{H}_{2} \mathrm{O}$, entsprechend $1,45 \% \mathrm{H}$ und $0,0915 \mathrm{CO}_{2}$, entsprechend $14,17 \% \mathrm{C}$.
Gefunden:
$\mathrm{H}=1, \mathbf{4 5} \%$
$\mathrm{C}=14,17 \%$
Berechnet auf $\mathrm{C}_{4} \mathrm{H}_{3} \mathrm{O}_{5} \mathrm{Bi}$ :

$$
\mathrm{H}=0,88 \%
$$$$
\mathrm{C}=14,15 \% \text {. }
$$

Da das in der Kälte dargestellte Wismutlaktat in Wasser sehr leicht löslich war und unter Zusatz von Milchsäure beim Kochen 
in unlösliches Wismutlaktat übergeführt nerden konnte, war anzunehmen, daB man auch durch Umsetzung des löslichen Wismutlaktats mit überschüssiger Apfelsäure zu einer apfelsauren Wismutverbindung gelangen konnte. Die Annahmen beatätigten sich, und ich stellte ein Wismutmalat dar, indem ich das in Wasser gelöste freie Milchsüure enthaltende Wismutlaktat mit einer wässerigen Apfelsäurelösung im Uoberschuß versetzte und darauf die Mischung bis zum Sieden erhitzte. Man konnte darauf ein Ausfallen eines ueißen krystallinischen Körpers wahrnchmen, dessen Eigenschaften und Zusammensetzung mit dem eingangs boschriebenen apfelsauren Wismut übereinstimmen. Nach vollkommenem Ausfallen des neuen Körpers brachte man deruselben auf ein Filter und wusoh gut mit Wasser aus. Man kann die Umsetzung auch in der Kälte vornehmen, nur resultiert dabei ein weniger schönes krystallinisches Produkt.

Die nachstehenden Analysen ergaben die Identität mit dem zuerst beschriebenen Wismutinalat.

a) Krystallwasser-Bestim mung.

1. $0,3913 \mathrm{~g}$ gaben beim Trocknen bei $105^{\circ} 0,0208 \mathrm{H}_{2} \mathrm{O}$ ab, entsprechend $5,31 \%$.

2. $0,3714 \mathrm{~g}$ gsben beim Trocknen bei $105^{\circ} 0,0194 \mathrm{H}_{2} \mathrm{O} \mathrm{ab}$, entsprechend $5,22 \%$.

3. $0,3851 \mathrm{~g}$ gaben beim Trocknen bei $105^{\circ} 0,0197 \mathrm{H}_{2} \mathrm{O}$ ab, entsprechend $5,11 \%$.

4. $0,4940 \mathrm{~g}$ gaben beim Trocknen bei $105^{\circ} 0,0260 \mathrm{H}_{2} \mathrm{O}$ ab, entBprechend $5,26 \%$.

5. $0,5128 \mathrm{~g}$ gaben beim Trocknon bei $105^{\circ} 0,0280 \mathrm{H}_{2} \mathrm{O} \mathrm{ab}$, entsprechond $5,07 \%$.

6. $0,6145 \mathrm{~g}$ gaben beim Trocknen bei $105^{\circ} 0,0263 \mathrm{H}_{2} \mathrm{O}$ ab, ontsprechend $5,11 \%$

b) Wismutbestimmung als $\mathrm{Bi}_{2} \mathrm{~S}_{3}$ und $\mathrm{Bi}_{2} \mathrm{O}_{3}$.

1. 0,3654 wasserfreie Substunz gaben $0,277 \mathrm{Bi}_{2} \mathrm{~S}_{3}$, onteprechend $61,58 \% \mathrm{Bi}$.

2. 0,4080 gaben $0,3564 \mathrm{Bi}_{2} \mathrm{~S}_{3}$, entsprechend $61,87 \% \mathrm{Oi}$.

3. 0,4861 wasserfreie Substanz gaben $0,3311 \mathrm{Bi}_{2} \mathrm{O}_{3}$, entsprechend $61,08 \% \mathrm{Bi}$.

4. 0,4872 gaben $0,3315 \mathrm{Bi}_{2} \mathrm{O}_{3}$, enteprechend $61,02 \% \mathrm{Bi}$.

c) Elementaranalyse.

0,1738 wasserfreies Salz gaben $0,0180 \mathrm{H}_{2}($ ), entoprecherd 1, 15:0 $\mathrm{H}$ und $0,0920 \mathrm{CO}_{2}$, entsprechend 14,43\% $\mathrm{C}$.

0,1705 wasserfreies Salz gaben $0,0210 \mathrm{H}_{2} \mathrm{O}$, entsprechend $1,36 \% \mathrm{H}$ und $0,0803 \mathrm{CO}_{2}$, entsprechend $14,44 \% \mathrm{C}$. 
Gofunden:

$\mathbf{H}_{\mathbf{\gamma}} \mathbf{O}=\mathbf{5 , 0 7 \%}$
$\mathbf{B i}=\boldsymbol{\beta} \mathbf{1 , 5 8 \%}$
$\mathbf{H}=1,15 \%$
$\mathbf{C}=14,43 \%$

Berechnet auf $\mathrm{C}_{4} \mathrm{H}_{3} \mathrm{O}_{8} \mathrm{Bi}+1 \mathrm{H}_{2} \mathrm{O}$ :

$$
\begin{aligned}
& \mathrm{H}_{2} \mathrm{O}=\mathbf{5 , 0 4 \%} \\
& \mathrm{Bi}=61,35 \% \\
& \mathrm{H}=0,88 \% \\
& \mathrm{C}=14,15 \%
\end{aligned}
$$

\section{Vorbladungen dos Whomuts ait der Woloedure.}

Der erste, der eine Wismutweinsäureverbindung beschrieben hat, ist $\mathrm{Sch}$ warze $\mathrm{n} \mathrm{ber}^{\mathbf{1}}$ ). Er erhielt im Jahre 1847 einen weißen krystallinischen Körper, indem er Wismutoxyd - erhalten durch Digerieren von Wismutnitrat mit Aetznatron - mit Weinstein kochte. Bei dieser Manipulation muß aber Wismutoxyd im UeberschuB vorhanden sein. Das klare Filtrat wurde auf dem Wassetbade konzentriert, wobei sich ein Krystallpulver abschied, welohes $\mathrm{Sch}$ warze $\mathrm{nberg}$ als Wismutbrechweinstein von der Formel $\mathrm{C}_{4} \mathrm{H}_{2} \mathrm{O}_{6} \mathrm{KBi}$ ansprach. $\mathrm{R}$. Sch neider) erhielt aus einer heißen Mischung einer mäßig konzentrierten Lösung von 5 Teilen Wismutoxyd in Salpetersäure mit einer konzentrierten Lösung von 4 Teilen Weinsäure in Wasser beim Abkühlen kleine glïnzende Krystalle und stellte für den neuen Körper die Formel $\mathrm{Bi}_{2}\left(\mathrm{C}_{4} \mathrm{H}_{4} \mathrm{O}_{4}\right)_{3} .6 \mathrm{H}_{2} \mathrm{O}$ auf, welche einem neutralen Wismuttartrat entsprechen würde. In einer längeren Studie bcrichtet $B$ a u d r a n? unter anderem auch über Verbindungen der Weinsäure und ihrer Kaliumsalze mit dem Wismut. Am Anfang dieser Arbeit erwähnte ich bereits, daß $B$ a u d ra n zwei verschiedene Wismuthydroxyde erhalten haben will, je nachdem man das Hydroxyd durch Fällen einer Wismutlösung mit Alkali oder durch Eingießen einer Wismutlösung in das Alkali darstellte. Nun bohauptet $B$ a u d r a n, daß es ihm mit großer Leichtigkeit gelungen sei von diesen zwei verschiedenen Hydroxyden auch zwei verschiedene Wismutweinsäureverbindungen darzustellen.

Aus frisch gefälltem Wismuthydroxyd, welches durch Eintragen einer Wismutlösung in überschüssiges Alkali bei gewölınlicher Temperatur gewonnen worden war, erhielt er durch Finwirkung eines Moleküls W'einsäure die freie Wismutweinsäure von der Formel $\mathrm{BiOC}_{4} \mathrm{H}_{6} \mathrm{O}_{6}+\mathrm{H}_{2} \mathrm{O}$, welche sich in 228 Teilen Wasser lösen sollte, in kleinen prismatischen Nadeln, die durch Alkali nicht zersetzt werden; setzte er dieser alkalischen Lösung die berechnete

1) S ch w a r e n berg, Liebig's Annal. 61, 224.

ग) R. S o h n o i d er, Liebig's Annal. 88, 260.

ग) $B$ a udran, l. c. 
Menge Kalilauge zu, so soll der Wismutbreohweinstein von der Formel $\mathrm{K}(\mathrm{BiO}) \mathrm{C}_{4} \mathrm{H}_{4} \mathrm{O}_{6}+\mathrm{H}_{2} \mathrm{O}$ auskrjstallisieren. Die alkalische Lösung obiger Wismutweinsäure wird weder warm noch kalt durch Wasser getrübt.

Versetzt man durch das Eintragen von Alkali zu einer Wismutlösung gewonnenes Wismuthydroxyd mit einer Kaliumbitartratlösung, so fält nach B a u d $\mathrm{s}$ n's Angaben nach kurzer Zeit ein krystallinischer Körper aus von der Formel $\mathrm{Bi}\left(\mathrm{C}_{4} \mathrm{H}_{4} \mathrm{KO}_{3}\right)_{3}+3 \mathrm{H}_{2} \mathrm{O}$. Derselbe soll im Gegensatz zu dem Wismutbrechweinstein, der sich in 80 Teilen Wasser jöst, schon in 13 Teilen Wasser löslich sein. Eine freie Wismutweineäure hat $\mathrm{B}$ a u $\mathrm{d}$ r a n von diesem Wismuthydroxyd nicht dargestellt.

In neuester Zeit haben $A$. R o s e $n$ h eim und W. Vogela a $\mathrm{ng}^{1}$ ) die Wismuttartrate und Alkaliwismuttartrate neu bearbeitet, die vorhandenen Untersuchungsergebnisse nachgeprüft und sind $\mathrm{zu}$ folgenden Resultaten gekommen. - Im Gegensatz zu den Angaben von $\mathrm{S}$ c h w a $\mathrm{r} z$ on berg und $\mathrm{B}$ a u d r a n konnten diese Autoren einen Wismutbrechweinstein nicht erhalten. Im übrigen berichten dieselben auch, daß die Angaben von $\mathrm{B}$ a u d r a $\mathrm{n}$ mit großer Vorsicht aufgenommen werden müssen. - Die nach der S chneider'schen Methode dargestellte Wismutweinsäure enthält nach $R$ osenheim und Vogelsang Salpetersäure. Ich kann diese Angabe durch meine eigenen Versuche bestätigen. Es lag sonach ein Wismuttartratnitrat vor, dem $R$ ose $n$ h ei m und $\mathrm{V}$ ogelsang die Formel $\mathrm{Bi}\left(\mathrm{C}_{4} \mathrm{H}_{4} \mathrm{O}_{6}\right) \mathrm{N} . \mathrm{O}_{3} .5 \mathrm{H}_{2} \mathrm{O}$ gaben. Um $z u$ einem salpetersäurefreien Wismuttartrat zu gelangen, wurde das $\mathrm{Sch} n \mathrm{e}$ i $\mathrm{d}$ er'sche Salz in kleinen Portionen in eine siedende konzentrierte Weinsäurelösung eingetragen, dabei ging das Salz langsam in Lösung und es schied sich nach dem Abkühlen ein salpetersäurefreies weinsaures Wismut in schön glänzenden Krystallen ab. Der erhaltene Körper löste sich klar in Alkali und wurde durch Wasser unter Abscheidung eines basischen Salzes zersetzt. Seine Formel wird als $\mathrm{Bi}\left(\mathrm{C}_{4} \mathrm{H}_{4} \mathrm{O}_{6}\right) \mathrm{C}_{4} \mathrm{H}_{5} \mathrm{O}_{8}+2 \mathrm{H}_{2} \mathrm{O}$ angegeben.

Körper der Zusammensetzung, wie sie $\mathrm{S}$ ch wa r ze n be rg und $\mathrm{B}$ a udran beschrieben haben, konnten $\mathrm{R}$ osen he im und Vogelsang nicht erhalten. Auch bei der Darstellung von Alkaliwismuttartraten bestätigen diese Autoren die Angaben der früheren Untersucher nicht. Von Salzen der Wismutweinsäure sind

1) A. Rosen heim und $\mathrm{V}$ ogelsang, Zeitsohr. f. anorgan. Chemie 48, 205, 1906. 
die Kalium-, Natrium- und Ammoniumverbindungen bereitet worden.

Natriumverbindungen der Wismutweinsäure konnten in krystallinischem Zustande nicht erhalten werden; da die alkalischen Lösungen ohne zu krystallisieren zu sirupösen Flüssigkeiten verdunsteten.

Was meine eigenen Untersuchungen über die Wismutweinsäure betrifft, so habe ich mich zuerst der Nachprüfung der Schneider'schen und Ba udran'schen Methode unterzogen und dann einen neuen Weg, der später beschrieben wird, zur Herstellung einer Wismutweinsäureverbindung durch Umsetzung des bereits erwähnten löslichen Wismutlaktats mit Weinsäure gefunden. Dann bringe ich den Beweis, da $B$ die beiden auf verschiedenem Wege gewonnenen Präparate miteinander identisch sind. Ferner habe ich versucht, krystallinische Natrium- und Kaliumsalze zu gewinnen, leider ohne Erfolg.

Zuerst stellte ich die Wismutweinsäure nach dem S c h n e id e r'schen Verfahren her, konnte aber nur die Angaben von Rosenheim und Vogelsang bestätigen, da auch ich Salpetersäure in dem Produkte nachwies. Da mir die von Bosenheim und Vogelsang vorgeschlagene Methode zu umständlich erschien, betrat ich den $B$ a u $\mathrm{dr}$ a n'schen Weg und ließ 20,0 frisch gefälltes Wismuthydroxyd, welches mit wenig Wasser innig verrührt war, mit einer konzentrierten wässerigen Lösung von 20,0 Weinsäure 6 Stunden aufeinander kalt einwirken. Nach dieser Zeit war die Masse zu einem Brei aus feinen Krystallnadeln erstarrt. Nach dem Absaugen und Entfernung der überschüssigen Weinsäure durch Auswaschen mit Alkohol, erhielt ich ein weißes Krystallmehl, das in Wasser kaum löslich war, von kohlensaurem Natrium und Aetzkali aber zu einer klaren Lösung aufgenommen wurde. Da B a u d r a n's Säure in 228 Teilen Wasser löslich sein soll, so mußte ich annehmen, daß ich eine andere Verbindung als $\mathrm{B}$ a u d ra n erhalten hatte. Die über Schwefelsäure getrockneten Krystalle nahmen bei $105^{\circ}$ im Mittel 9,8\% Wasser ab.

1: 0,7630 lufttrockenes Salz gaben bei $105^{\circ} 0,0725 \mathrm{H}_{2} \mathrm{O} \mathrm{ab}$, entsprechend $9,5 \%$.

2. 0,6884 lufttrockenes Salz gaben bei $105^{\circ} 0,0654 \mathrm{H}_{2} \mathrm{O}$ ab, entsprechend $9,5 \%$.

3. 0,8980 lufttrockenes Salz gaben bei $105^{\circ} 0,0890 \mathrm{H}_{2} \mathrm{O}$ ab, entsprechend $9,91 \%$.

4. 0,5584 lufttrockenes $\mathrm{Salz}$ gaben bei $105^{\circ} 0,0549 \mathrm{H}_{2} \mathrm{O}$ ab, entsprechend $9,83 \%$. 
Das Wismut bestimmte ich in der bereits angegebenen Weise als Oxyd. Die Analysenresultate lasse ich folgen.

1. 0,7689 wasserfreie Substanz gaben $0,3578 \mathrm{Bi}_{2} \mathrm{O}_{3}$, entsprechend $41,73 \% \mathrm{Bi}$.

2. 0,7640 Substanz gaben $0,3562 \mathrm{Bi}_{2} \mathrm{O}_{3}$, entsprechend $41,81 \% \mathrm{Bi}$.

Die Elementaranalyse gab folgende Zahlen:

1. 0,1762 wasserfreie Substanz gaben $0,0310 \mathrm{H}_{2} \mathrm{O}$, entsprechend $1,95 \% \mathrm{H}$ und $0,1245 \mathrm{CO}_{2}$, entsprechend $18,27 \% \mathrm{C}$.

2. 0,1680 wasserfreie Substanz gaben $0,0305 \mathrm{H}_{2} \mathrm{O}$, entsprechend $1,99 \% \mathrm{H}$ und $0,1180 \mathrm{CO}_{2}$, entsprechend $19,15 \% \mathrm{C}$.

Zur Kontrolle habe ich auch die wasserhaltige und über Schwefelsäure getrocknete Substanz verbrannt und bin zu folgenden Zahlen gekommen:

1. 0,1713 wasserhaltiges Salz gaben $0,0385 \mathrm{H}_{2} \mathrm{O}$, entsprechend $2,49 \% \mathrm{H}$ und $0,1104 \mathrm{CO}_{2}$, entsprechend $17,57 \% \mathrm{C}$.

2. 0,1725 wasserhaltige Substanz gaben $0,0450 \mathrm{H}_{2} \mathrm{O}$, entsprechond $2,89 \% \mathrm{H}$ und $0,1097 \mathrm{CO}_{2}$, entsprechend $17,34 \% \mathrm{C}$.

Die Resultate der Analysen führen zu der Annahme, daß ein Atom Wismut sich mit zwei Molekülen Weinsäure zu einem sauren Salze (Wismutweinsäure) von der Formel $\mathrm{C}_{8} \mathrm{H}_{9} \mathrm{O}_{12} \mathrm{Bi}+3 \mathrm{H}_{2} \mathrm{O}$ verbinden.

Gefunden:

$\mathrm{H}=1,95 \%$

$\mathrm{C}=19,15 \%$

$\mathrm{Bi}=41,72 \%$
Berechnet auf $\mathrm{C}_{8} \mathrm{H}_{9} \mathrm{O}_{12} \mathrm{Bi}$ was erf rei :

$\mathrm{H}=1,78 \%$

$\mathrm{C}=18,89 \%$

$\mathrm{Bi}=41,24 \%$.

Wasserhaltige substanz.

Gefunden:

$$
\begin{aligned}
& \mathrm{H}_{2} \mathrm{O}=9,5 \% \\
& \mathbf{H}=2,89 \% \\
& \mathrm{C}=17,34 \%
\end{aligned}
$$

Berechnet auf $\mathrm{C}_{8} \mathrm{H}_{8} \mathrm{O}_{12} \mathrm{Bi}+3 \mathrm{H}_{2} \mathrm{O}$ :

$$
\begin{aligned}
& \mathrm{H}_{2} \mathrm{O}=9,65 \% \\
& \mathrm{H}=2,68 \% \\
& \mathrm{C}=17,15 \% .
\end{aligned}
$$

Die von mir dargestellte Verbindung unterscheidet sich von der Wismutweinsäure des $\mathrm{R}$ os e $\mathrm{n}$ h e i m und V o g e ls a ng nur um ein Molekül höheren Krystallwassergehaltes.

Ein andercr Weg, auf welchem ich zu der Wismutweinsäure gelangte, bestand darin, daß ich das in Wasser leicht lösliche Wismutdilaktat mit einem Ueberschuß von wässeriger Weinsäurelösung versetzte und diese Mischung bis zum Sieden erhitzte. Beim Abkühlen der anfangs klaren Flüssigkeit krystallisierte die Wismut- 
weinsäure in prä̀htigen prismatischen Nadeln aus; dieselbe löste sich glatt ohne jegliche Trübung unter Aufbrausen in koldensaurem Natron und Alkalilauge. Im übrigen hatte sie dieselben Eigenschaften wie die vorher beschriebene Säure, nur war das Präparat schöner krystallinisch ausgebildet. Nachdem die Weinsäure zuerst wit Wasser, später mit Alkohol ausgewaschen war, analysierte ich die Substanz und bin dabei zu denselben Resultaten gekommen, wie bei der zuerst dargestellten Wismutweinsäure.

Krystallwasserbesti m mugen. $9,98 \%$.

1. $0,643 \theta$ Salz nahmen bei $105^{\circ} 0,0643 \mathrm{H}_{2} \mathrm{O} \mathrm{ab}$, enteprechend $9,91 \%$.

2. 0,6082 Salz nahmen bei $105^{\circ} 0,0603 \mathrm{H}_{2} \mathrm{O}$ ab, entsprechend

Wis m u t best i m m ung.

I. 0,5796 wasserfreie Substanz gaben $0,2681 \mathrm{Bi}_{2} \mathrm{O}_{3}$, enteprechend $+1,47 \% \mathrm{Bi}$.

2. 0,6469 wasserfreie Substanz gaben $0,2624 \mathrm{Bi}_{2} \mathrm{O}_{3}$, entsprechend $+1,38 \% \mathrm{Bi}$.

\section{Eleine ntaranalyse.}

1. 0,1520 wasserfreie Substanz gaben $0,0270 \mathrm{H}_{2} \mathrm{O}$, entsprechend $1,97 \% \mathrm{H}$ und $0,1055 \mathrm{CO}_{2}$, entsprechend $18,92 \% \mathrm{C}$.

2. 0,1563 Substanz gaben $0,0285 \mathrm{H}_{2} \mathrm{O}$, entsprechend $2,02 \% \mathrm{H}$ und $0,1085 \mathrm{CO}_{2}$, entsprechend $18,93 \% \mathrm{C}$.

$$
\text { Gefunden: }
$$

Kirystallwasser $=9,91 \%$

$$
\begin{aligned}
& \mathrm{H}=1,97 \% \\
& \mathrm{C}=18,93 \% \\
& \mathrm{Bi}=41,38 \%
\end{aligned}
$$

Berechnet auf $\mathrm{C}_{8} \mathrm{H}_{2} \mathrm{O}_{12} \mathrm{Bi}+3 \mathrm{H}_{2} \mathrm{O}$ :

$\begin{aligned} \text { Lrybtallwasser } & =9,65 \% \\ \mathbf{H} & =1,78 \% \\ \mathbf{C} & =18,99 \% \\ \mathrm{BI} & =41,24 \%\end{aligned}$

Da sich die Wigmutweinsäure in Natronkarbonat unter Aufbrausen klar löste, so konnte man hoffen, durch Sättigen einer Satronkarbonatlösung mit Wismutweinsäure und darauffolgendem Eindarapfon ein Natriumdoppelsalz zu erhalten. Es resultierten aber nach dem Eindampfen sirupöste, nach dem Trocknen beioht zu einen feinen weißen Pulver zerreibliche Mraseon, bei deren analytiacher Untersuchung ioh bis jetzt nicht zu konstanten Werten für Natrium oder Kalium gelangen konnte. Das Natriundoppelsalz dürfte trotzdem für alle diejenigen Fälle brauchbar sein, wo neutrale wässerige Wismutlösungen zu medizinischen Zwecken erforderlich sind. 


\section{Verbindungen des Wlamuts mit der Zltronenelure.}

Obgleich sich meine Untersuchungen über zitronensaures Wismut im wesentlichen auf Nachprüfungen der vorhandenen Ergebnisse beziehen, und ich nur die Darstellung des Wismutcitrats durch Umsetzung des löslichen Wismutlaktats durch Zitronensäure als neu bezeichnen kann, so will ich dennoch als Abschluß dieser Arbeit einiges über zitronensaures Wismut berichten.

R. R o t $h$ e $\mathrm{r}^{1}$ ) bereitet ein krystallinisohes zitronensaures Wismut, indem er 10,0 Magisterium Bismutii mit einer lösung von 7,0 Zitronensäure in 30,0-40,0 Wasser einige Minuten erhitzt, bis ein Tropfen der Mischung mit Ammoniakwasser eine klare Lösung gibt. Die krystallinische Masse wird dann mit dem 8-10 fachen Volumen verdünnt und nach einiger Zeit dekantiert; darauf gut ausgewaschen und getrocknet. Das Salz entspricht der Formel $\mathrm{C}_{6} \mathrm{H}_{5} \mathrm{O}_{7} \mathrm{Bi}$. Löst man das zitronensaure Wismut unter gelindem Erwärmen in wässerigem Ammoniak und läßt es erkalten, so erbält man eine krystallinische Masse von der Zusammensetzung $\left(\mathrm{NH}_{4}\right)_{3} \mathrm{C}_{6} \mathrm{H}_{5} \mathrm{O}_{7} \mathrm{Bi}(\mathrm{OH})_{3}$.

Fast dieselbe Darstellungsweise schreibt die Pharmakopöe der Vereinigten Staaten von Nordamerika vor, in welcher das Wismutcitrat offizinell ist. Hier werden 100,0 Wismutsubnitrat mit einer Lösung von 70,0 Zitronensäure in 400,0 Wasser 15 Minuten lang gekocht oder bis ein Tropfen des Gemisches mit Ammoniakwasser eine klare Lösung gibt. Dann fügt man 5000,0 Wasser zu, läßt absetzen und wäscht den Niederschlag zunächst durch Dekantieren, dann auf dem Filter aus, bis der Ablauf geschmacklos ist. Das. Präparat ist ein geruch- und geschmackloses, amorphes oder mikrokrystallinisches Pulver.

Zu demselben Wismutcitrat - nur von besserer krystallinischer Form, es resultieren kleine sphäroidisohe Wärzchen - gelangt man, wenn man 8,0 Zitronensëure in wenig Wasser gelöst mit 10,0 frisch gefälltem Wismutoxyd 5 Minuten lang kocht, oder bis eine kleine Probe sich in Ammoniakwasser klar löst. Das krystallinische Gemisch wird hierauf zuerst mit Wasser dekantiert, auf ein Filter gebracht und mit Alkohol nachgewaschen. Die Analysen bestätigen, daB das normale zitronensaure Wismut von der Formel $\mathrm{C}_{6} \mathrm{H}_{5} \mathrm{O}_{7} \mathrm{Bi}$ vorliegt. Das Wismut mußte ich in dieser Verbindung als sulfid bestimmen, weil das Wismutcitrat beim Erhitzen über der Flamme

1) R. R ot her, Pharm. Journ. Trans. (3), 6, 764. 
flüchtig war. Krystallwasser gab das Salz beim Trocknen bei $105^{\circ}$ nicht ab.

1. 0,5473 substenn geben 0,3646 $\mathrm{Bi}, \mathbf{s}_{2}$, enteptechend $52,62 \% \mathrm{Bi}$.

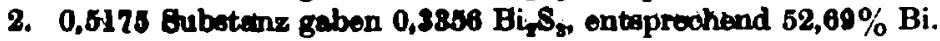

Die Elementaranalyse ergab:

0,1862 lufttrockenes Salz gaben $0,0270 \mathrm{H}_{2} \mathrm{O}$, entsprechend $1,61 \% \mathrm{H}$ und $0,1220 \mathrm{CO}_{2}$, entsprechend $17,86 \% \mathrm{C}$.
Gefunden:
$\mathbf{H}=1,61 \%$
Berechnet auf $\mathrm{C}_{6} \mathrm{H}_{6} \mathrm{O}_{7} \mathrm{Bi}$ :
$\mathrm{C}=17,88 \%$
$\mathbf{H}=1,25 \%$
$\mathrm{C}=18,13 \%$
$\mathrm{Bi}=52,62 \%$
$\mathrm{Bi}=62,39 \%$.

Natürlich konnte man das zitronensaure Wismut auch durch Umsetzung des löslichen Wismutlaktats mittels Zitronensäure gewinnen. Ioh löste das milchsaure Wismut in Wasser, setzte demselben eine konzentrierte wässerige Lösung von Zitronensäure zu und erhitzte das Gemisch bis zum Sieden. Beim Abkühlen setzten sich dann wetzsteinförmige Krystalle ab. Der gewonnene Niederschlag wird zuerat mit Wasser delsantiert und zuletzt auf einem Filter mit Alkohol ausgewaschen. Die Analysen bestätigen, daß auch hier das normale Wismutcitrat entstanden ist.

1. 0,4922 Bubetanz gaben $0,3165 \mathrm{Bi}_{2} \mathrm{~B}_{2}$, entaprechend $52,44 \% \mathrm{Bi}$.

2. 0,4902 Bubstane gaben $0,3155 \mathrm{Bi}_{2} \mathrm{~B}_{2}$, entsprechend $62,49 \% \mathrm{Bi}$.

1. 0,1828 Substanz gaben bei der Elementaranalyse 0,0285 $\mathrm{H}_{2} \mathrm{O}$, enteprechend $1,73 \% \mathrm{H}$ und $0,1238 \mathrm{CO}$, enteprechend $18,47 \% \mathrm{C}$.

2. 0,1962 Substanz gaben $0,0290 \mathrm{H}_{8} \mathrm{O}$, enteprechend $1,64 \% \mathrm{H}$ und $0,1304 \mathrm{CO}_{2}$, enteprechend $18,12 \% \mathrm{C}$.

$$
\begin{aligned}
& \text { Gefunden: } \\
& \mathbf{H}=1,64 \% \\
& \mathbf{C}=18,12 \% \\
& \mathbf{B i}=52,29 \%
\end{aligned}
$$

Bereohnet an $\mathrm{C}_{9} \mathrm{H}_{8} \mathrm{O}_{7} \mathrm{Bi}$ :

$$
\begin{aligned}
& \mathbf{H}=1,25 \% \\
& \mathbf{C}=18,13 \% \\
& \mathrm{Bi}=62,39 \% .
\end{aligned}
$$

Am Schlusse dieser Arbeit, welche im pharmakologischen Institut der Universität Leipzig ausgeführt nurde, ist mir Bedürfnis Herrn Geheimrat Profeesor Dr. R. B o e h m auch an diecor 8telle meinen herzlichsten Dank für die Anregung and gütigste Unterstïtıung bei der Ausführung dieser Arbeit auszuspreohen. 